\title{
Analysis of circulating miRNAs 21 and 375 as potential biomarkers for early diagnosis of prostate cancer
}

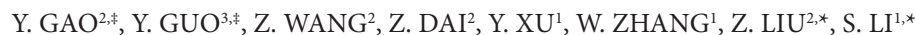

${ }^{1}$ Department of Biochemistry, Institute of Glycobiology, Dalian Medical University, 9 South Lvshun Road Western Section, Dalian 116044, Liaoning, China; ${ }^{2}$ Department of Urological Surgery, the Second affiliated hospital of Dalian Medical University, Dalian, Liaoning 116027, China; ${ }^{3}$ Department of Microecology, Dalian Medical University, Dalian, Liaoning 116044, China

*Correspondence: lisheng_1996@163.com; letter89@163.com

*Contributed equally to this work.

Received December 10, 2015 / Accepted March 23, 2016

\begin{abstract}
To investigate the diagnostic potential of plasma miR-21 and miR-375 by comparing their levels in prostate cancer (PCa) patients to subjects without cancer in a Chinese population. The study population included 57 PCa patients and 28 benign prostatic hyperplasia (BPH) patients. The plasma levels of miR-21 and miR-375 were quantitated with Taqman based quantitative real-time polymerase chain reaction. In the BPH group, the median relative expression levels of miR-21 and miR-375 were 0.07 and 0.55 , respectively; In the PCa group, the median values of miR-21 and miR-375 were 1.32 and 1.74, respectively. Both miR-21 ( $\mathrm{p}=0.014)$ and miR-375 ( $\mathrm{p}=0.005)$ plasma levels were significantly higher in PCa group than in BPH group. Using ROC analysis the AUC of miR-21 was $0.799,95 \%$ CI between 0.690 and $0.908\left(\mathrm{p}=8 \times 10^{-6}\right)$; for miR-375, the AUC was $0.757,95 \%$ CI between 0.640-0.874 ( $\mathrm{p}=0.000126)$. The largest AUC $(0.881)$ was obtained when the data of miR-21, miR-375 and PSA were combined, with a sensitivity of $87.7 \%$ and a specificity of $75 \%$. The results of this study showed that circulating miR-21 and miR-375 can discriminate PCa patients from BPH controls at early stages. Combinations of the studied miRNAs and PSA remarkably increased specificity compared with PSA alone. The combination method has the potential to be used as a noninvasive diagnostic cocktail for PCa screening.
\end{abstract}

Key words: miR-21, miR-375, biomarker, prostate cancer, in vitro diagnosis

Prostate cancer $(\mathrm{PCa})$ is the most diagnosed male cancer and the second leading cause of cancer-related deaths in the Western world [1]. Along with the economic growth and change of life style, the incidence of PCa is remarkably increasing each year in East Asia, and the prevalence of prostate cancer in East Asian men is likely similar to that in Western male populations [2]. Although researchers are aiming persistently at inventing novel therapeutic approaches, it is still of primary importance to discover a powerful diagnostic method able to detect PCa at early stages. Since its approval by FDA in 1986, prostate-specific antigen (PSA) measurement has always been the mainstream noninvasive diagnostic methods for PCa. Together with digital rectal examination (DRE), and histopathological evaluation of prostate needle biopsies, PSA test have helped clinicians to diagnose PCa patients all around the world. However, PSA test has even significant disadvantages. The sensitivity is low and the optimal thresh- old for biopsy is unclear [3]. On the one hand many patients were over diagnosed due to negative positivity, on the other hand most patients (70\%) are diagnosed at the late stages of $\mathrm{PCa}$ and lose the best chances for early treatments [4]. Hence, there is more and more interest in searching for alternative noninvasive biomarkers such as miRNAs.

MiRNAs are a class of small noncoding RNAs that regulate about $60 \%$ of protein expression in the human genome [5], and have been found to play key roles in tumorigenesis. Some miRNAs are upregulated during cancerous development, rendering them as potential biomarkers for cancer diagnosis [6]. Recent studies have demonstrated that miRNAs can be exported out of cells and circulate in the blood. These miRNAs are relatively stable due to their short sequence difficult for RNases to attack. It has been reported that expression patterns of miRNAs can distinguish patients from healthy individuals and the expression characteristics are associated with the 
developmental lineage of the cancer and of the disease stage $[7,8]$. Circulating miRNAs have been evaluated as potential noninvasive biomarkers for PCa [4, 9-11]. However, the findings are not conclusive. There is still a need of more cohort studies for validation.

MiR-21 is an anti-apoptotic agent functioning via p53 network, especially by targeting programmed cell death 4 (PDCD4) and the phosphatase and tensin homologue (PTEN) mRNAs [12]. Over expression of miR-21 in a prostate cancer cell line ( $\mathrm{LnCaP}$ ) could stimulate androgen-dependent cell growth and rescue cells from androgen-deficient cell arrest [13]. MiR-375 has been reported to be involved in various types of tumorigenesis including hepatocellular carcinoma, gastric cancer as a tumor-suppressor [14]. Interestingly, miR-375 was found to be upregulated 9.1-fold in primary prostate carcinoma compared with normal prostate tissue using miRNA deep sequencing technology, though the mechanism is still unclear [15]. In the present study, we analyzed miR-21 and miR-375 levels in plasma of 57 PCa patients and 28 Benign prostatic hyperplasia (BPH) patients, and compared their AUC values with PSA test using ROC analysis, in order to evaluate their potential role as specific noninvasive molecular biomarkers for prostate cancer diagnosis.

\section{Patients and methods}

Patients and samples collection. The ethics committee of the Second Affiliated Hospital of Dalian Medical University approved this study, and written consent was obtained from all patients to provide information and samples for research purposes. From July 2014 to March 2015, 57 patients aged 5075 years and diagnosed as histologically confirmed primary PCa were enrolled in this study. Cases were excluded if they were diagnosed with other malignancies or previously had prostate surgery. 28 aged-matched men with lower urinary tract symptoms and diagnosed as benign prostatic hyperplasia were used as controls. Patients who were diagnosed as diabetes or had symptom of inflammation had been excluded before the study either.

Blood was withdrawn in ethylenediaminetetraacetic acid (EDTA) containing tubes and immediately centrifuged at $4{ }^{\circ} \mathrm{C}$ $1000 \mathrm{~g}$ for $20 \mathrm{~min}$. Plasma was collected and stored at $-80^{\circ} \mathrm{C}$ freezer until RNA purification.

RNA isolation and miRNA quantification. RNA was extracted from $800 \mu \mathrm{L}$ of plasma using miRNeasy serum/plasma kit (Qiagen, Product ID: 217184) according to manufacturer's instructions. The RNA was eluted in $50 \mu \mathrm{L}$ of nuclease free water, and 40 ng RNA (in $5 \mu \mathrm{L}$ ) was reverse transcribed with TaqMan $^{\oplus}$ MicroRNA Reverse Transcription Kit (Product ID: 4366597). cDNA was quantitated using Taqman based quantitative real-time polymerase chain reaction ( $\mathrm{qRT}-\mathrm{PCR}$ ) assay (Product ID: 4440040) specific for miR-21 (Primer product ID: 000397) and miR-375 (Primer product ID: 000564) according to manufacturer's recommended protocol on an Applied Bio- systems 7500 Sequence Detection System. Cycling conditions were $95^{\circ} \mathrm{C}$ for $10 \mathrm{~min}$ followed by 40 cycles at $95^{\circ} \mathrm{C}$ for $15 \mathrm{~s}$ and $60^{\circ} \mathrm{C}$ for $1 \mathrm{~min}$. All reactions were performed in triplicate. The expression levels of circulating miRNAs are presented as the threshold cycle $(\mathrm{Ct})$ values. Endogenous reference U6 was used as an internal control for normalizing the data. The comparative $\mathrm{Ct}(\Delta \mathrm{Ct})$ method was used to analyze the miRNA expression levels $\left(\Delta \mathrm{Ct}=\mathrm{Ct}_{\text {miRNA }}-\mathrm{Ct}_{\mathrm{U} 6}\right)$. Fold change between groups was calculated as the relative value $2^{-(\Delta \mathrm{CtPCa}-\Delta \mathrm{CtBPH})}$.

Statistical analysis. Since miRNA levels were skewed, $\log _{2}$ transformation was applied to make the data closely approximate normality in the final analysis. Statistical analysis between numerical variables of two groups were performed using unpaired Student's $t$ test for parametric data or MannWhitney rank sum test for nonparametric data. $P<0.05$ was considered as statistically significant.

Logistic regression was conducted to construct receiver operating characteristic (ROC) curves using expression level of miRNA adjusted for the matching factors (age, race). The area under the curve is calculated with $95 \%$ confidence interval. The specificity and sensitivity were identified by numerical integration of each ROC curve. A prediction model was built by fitting miRNAs into the logistic regression model, and the stepwise backward model was used to determine miRNA combinations in discriminating $\mathrm{PCa}$ patients with $\mathrm{BPH}$ patients. A likelihood ratio test $P<0.05$ was considered as statistically significant. All analyses were performed using SPSS 15.0 software.

\section{Results}

Statistical analysis of miR-21 and miR-375 relative expression. Clinicopathological characteristics of the patients enrolled in the study were listed in Table 1 . The median age of 57 PCa patients enrolled in this study was $73(60-85)$ years old, and that of $28 \mathrm{BPH}$ patients in this study was 72.45 (56-88). We compared the plasma levels of the two miRNAs between these two groups. In the BPH group, the median relative expression levels of miR-21 and miR- 375 were 0.07 and 0.55 , respectively; in the PCa group, the median values of miR-21 and miR-375 were 1.32 and 1.74 , respectively. Both miR-21 $(\mathrm{p}=0.014)$ and miR-375 ( $\mathrm{p}=0.005)$ plasma levels were significantly higher in PCa group than in BPH group (Figure 1, Table 2).

ROC curves were constructed to evaluate the potential power of miR-21 and miR-375 as invasive biomarkers (Figure 2). miR-21 exhibited the better discrimination between PCa patients and BPH control subjects than miR-375. The AUC of miR-21 was $0.799,95 \%$ CI between 0.690 and 0.908 $\left(p=8 \times 10^{-6}\right)$; For miR- 375 , the AUC was $0.757,95 \%$ CI between $0.640-0.874 \quad(p=0.000126)$. At the optimal cut-off value of $\mathrm{RQ}$, the sensitivity of miR-21 was $87.7 \%$ and specificity was $75.0 \%$. MiR-375 displayed a lower sensitivity of $75.4 \%$ and the same specificity of $75.0 \%$ compared with miR-21. PSA, the traditional biomarker for $\mathrm{PCa}$, exhibited the highest AUC of 0.833 in our study. With $6.9 \mathrm{ng} / \mathrm{ml}$ as the cut off value, PSA assay achieved the sensitivity of $91.2 \%$, higher than those of 
Table 1. Participants' Demographic and Clinical Characteristics

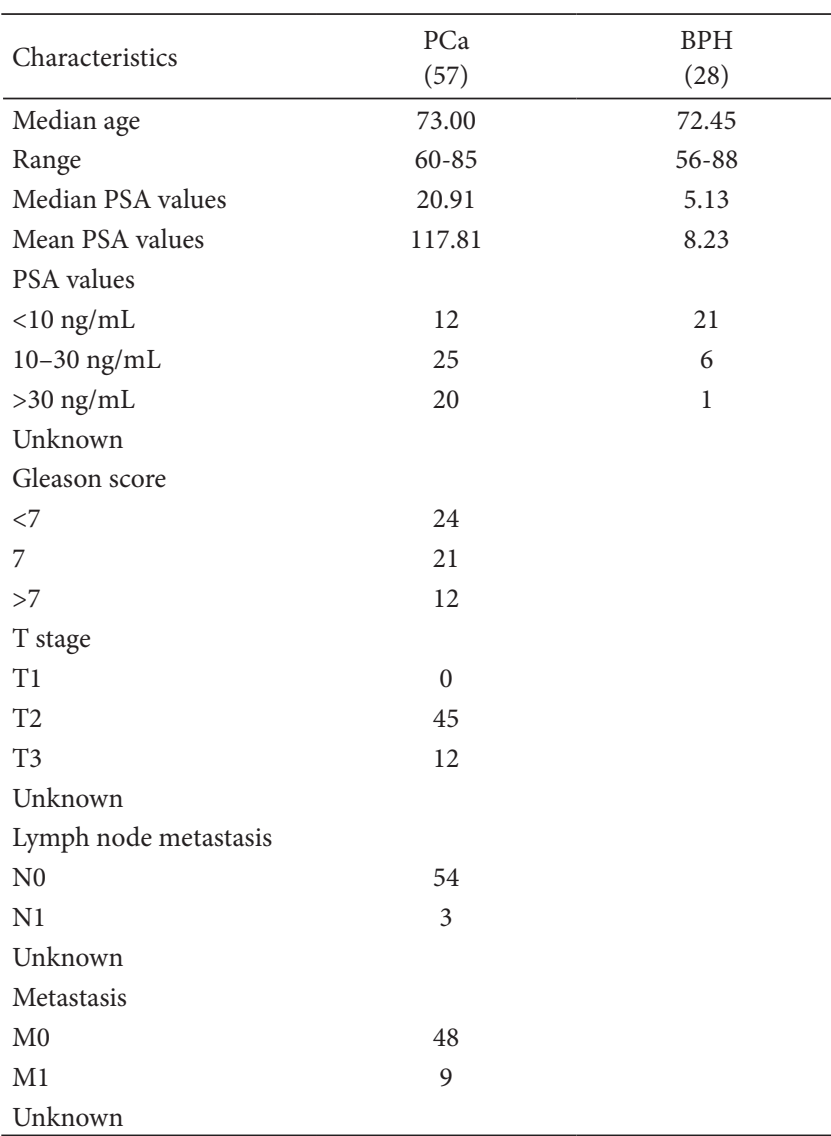

$\mathrm{PCa}$, prostate cancer; $\mathrm{BPH}$, benign prostatic hyperplasia; PSA, prostatespecific antigen
Table 2. Distribution of miR-21and miR-375 between PCa and BPH patients

\begin{tabular}{lcc}
\hline & PCa & BPH \\
\hline miR-21 & $0-92.68$ & $0-6.07$ \\
Range & 5.99 & 0.87 \\
Mean & 1.32 & 0.07 \\
Median & & \\
miR-375 & $0-47.46$ & $0-11.18$ \\
Range & 5.09 & 1.51 \\
Mean & 1.74 & 0.55 \\
Median &
\end{tabular}

miR-21 and miR-375; however, at the same time the specificity of PSA assay (67.9\%) was the lowest among the indices analyzed in the study.

The potential diagnostic ability of combinations of studied miRNAs and PSA. Spearman analysis was used to analyze the relationship between miR-21 and miR-375 expression. In the serum of PCa patients, a medium and significant correlation was found $\left(\mathrm{r}_{\mathrm{s}}=0.403, \mathrm{p}=0.002\right)$; while no significant correlation between miR-21 and miR-375 $\left(\mathrm{r}_{\mathrm{s}}=0.403, \mathrm{p}=0.228\right)$ was found in the group of BPH. In the serum of all subjects, miR-21 expression was weakly correlated with PSA $\left(r_{s}=0.320\right.$, $\mathrm{p}=0.003$ ), and miR-375 had no significant correlation with PSA $\left(r_{s}=0.139, p=0.206\right)$. A scatter graph was produced using SPSS software and shown in Figure 3.

Diagnostic accuracy affected by combinations of the studied miRNAs and PSA were analyzed with ROC curve (Figure 4).
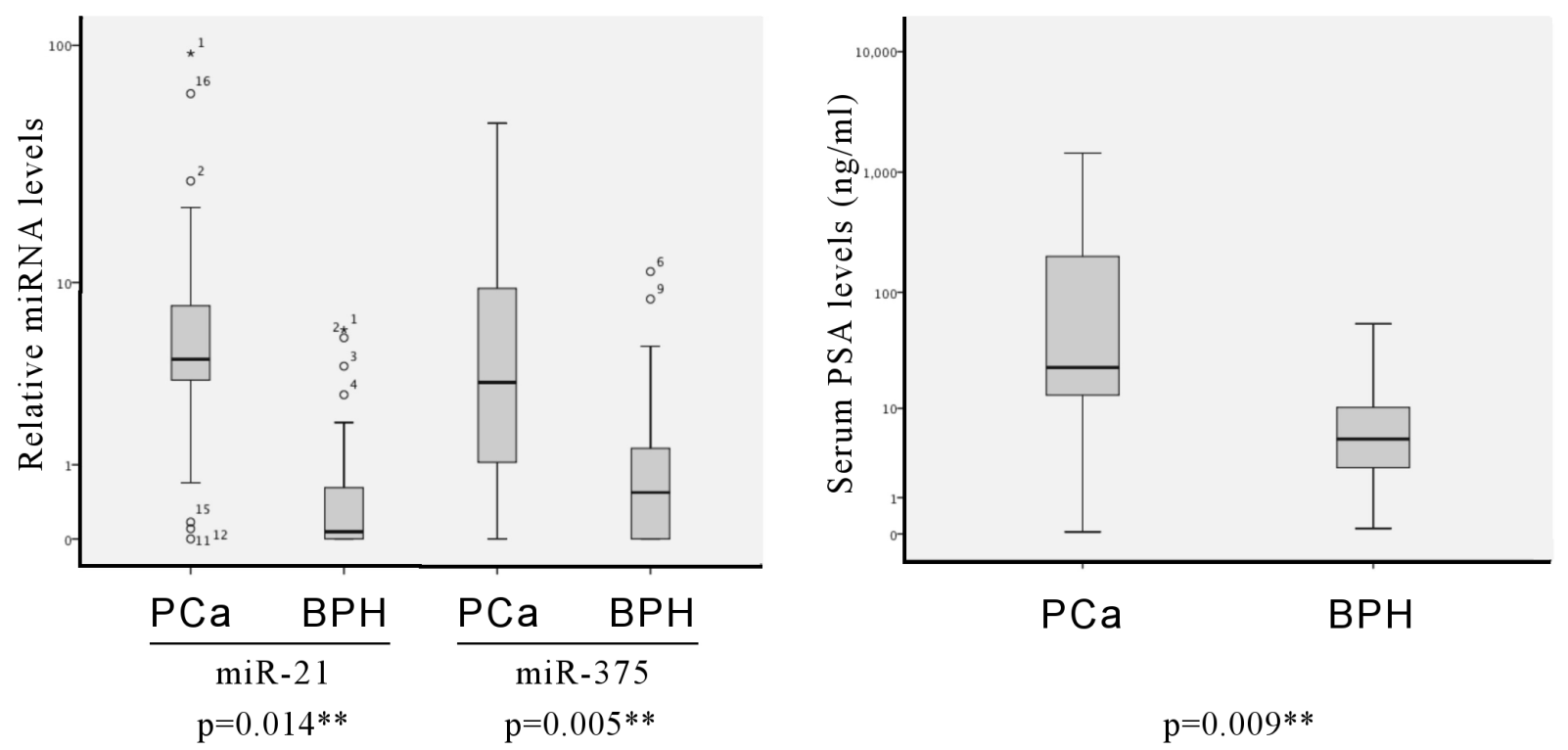

Figure 1. Box plots representing plasma miR-21 and miR-375 expression levels and serum PSA levels in prostate cancer (PCa) patients and benign prostatic hyperplasia (BPH) controls. Expression levels of the miRNAs (scale of y axis: log 10) are normalized to U6. BPH samples are used as calibrators. 

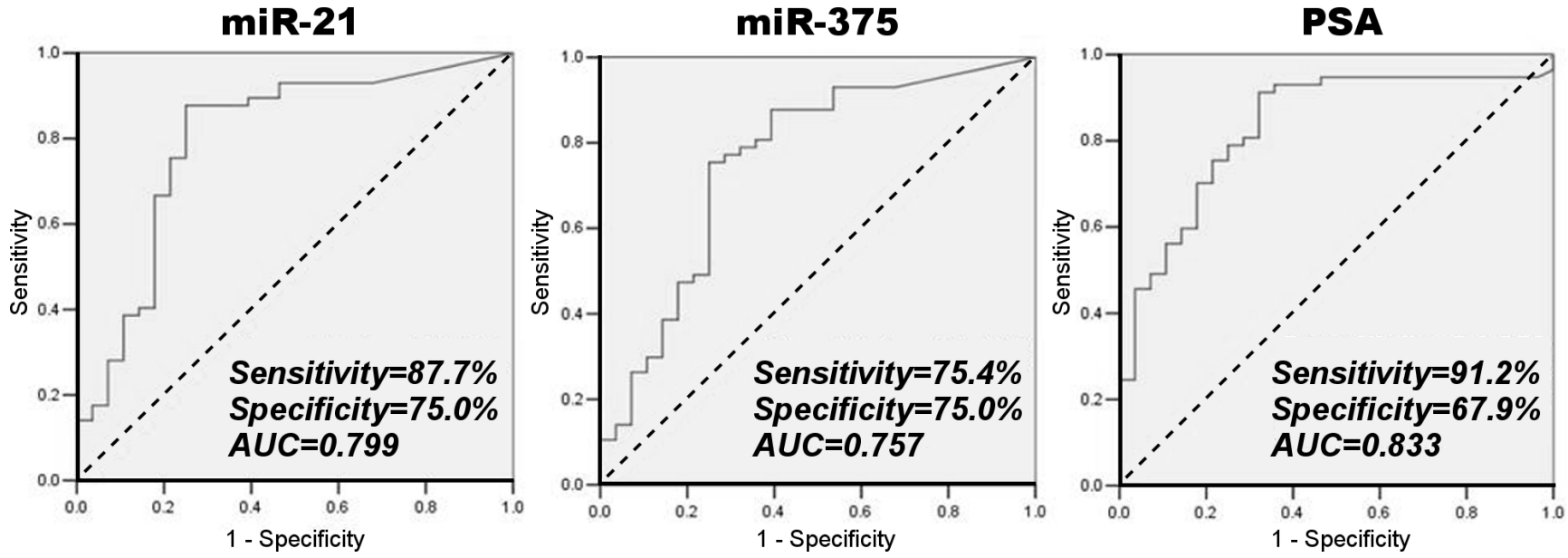

Figure 2. Receiver operating curve (ROC) curve analysis by using miR-21, miR-375 and prostate-specific antigen $(\mathrm{PSA})$ to differentiate PCa (n = 57) from BPH $(\mathbf{n}=28)$ controls.

It was found that combinations of the studied miRNAs and PSA remarkably increased specificity compared with PSA alone. The largest AUC (0.881) were achieved when all the three bio-molecules were combined.

In addition, we investigated whether miR-21 or miR-375 was significantly correlated with clinicopathological characteristics such as the Gleason score, tumor stage and development of lymph node metastasis. MiR-21 was correlated weakly with the Gleason score, while miR-375 was not (data not shown). To check whether the levels of the studied miRNAs were cor-

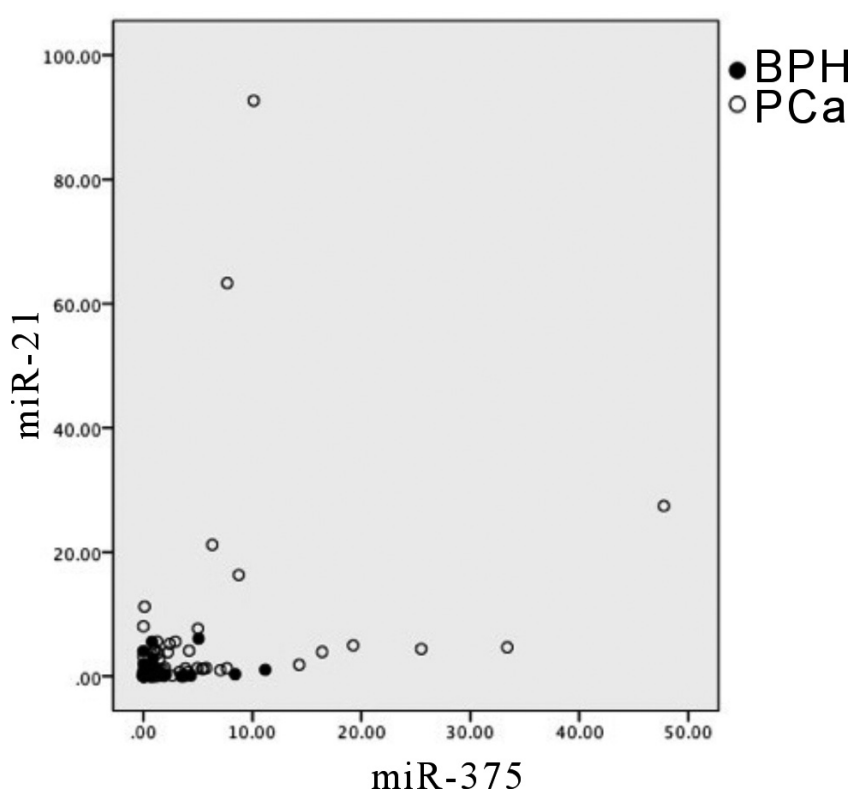

Figure 3. The relationship between miR-21 and miR-375 using a scatter graph is shown. Filled circles represent BPH patients, empty circles represent PCa patients. related with age, we performed the Spearman test appropriate for not normally distributed data. We did not observe statistically significant correlation between age and expression levels of the miR-21 and miR-375 within the PC patients and BPH subjects.

\section{Discussion}

The traditional PSA test for PCa is always criticized for its high false-positivity. Only about $25 \%$ of men receiving prostate biopsy due to an elevated PSA level actually have PCa [16]. Clinicians have always been looking for an alternative method. Since it was reported in 2008 for the first time that circulating miRNAs could distinguish PCa from healthy controls due to its abnormal expression level and remarkable stability in plasma [17], more than 100 miRNAs have been suggested as circulating biomarker candidates [16]. However, there is still much controversy over the performance of miRNAs in this issue. The purpose of the present study is to investigate the possibility of using circulating miR-21 and miR-375 expression as noninvasive biomarkers for early diagnosis of PCa.

We studied miR-21 and miR-375 expression in plasma of $57 \mathrm{PCa}$ patients and $28 \mathrm{BPH}$ patients. The median relative expression level of miR-21 in PCa group was 1.32, significantly higher than in BPH group (median: 0.07). This is consistent with the results of other research groups. In the study by Agaoglu et al., the expression of miR-21 in 51 patients with prostate cancer (median: 1.51) was significantly higher than 20 healthy controls (median: 0.039) [18]. Kotb et al. reported that miR-21 level in 10 PCa patients (median: 3.1) was significantly higher than $10 \mathrm{BPH}$ patients (0.175) [19].

In our study the median value of miR-375 in PCa group (1.74) was also significantly higher than in $\mathrm{BPH}$ group (median: 0.55). Wachet al. reported in 2015 that the expression of miR-375 in 146 PCa patients (median: 1.214) was 
miR-21, miR-375

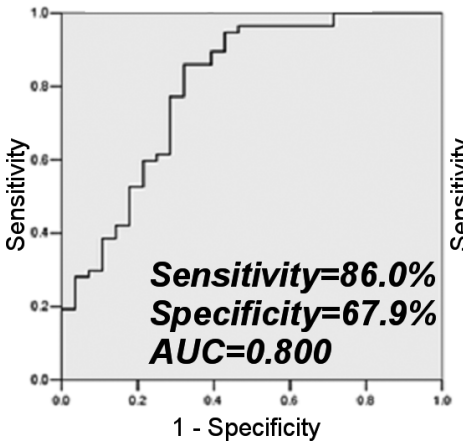

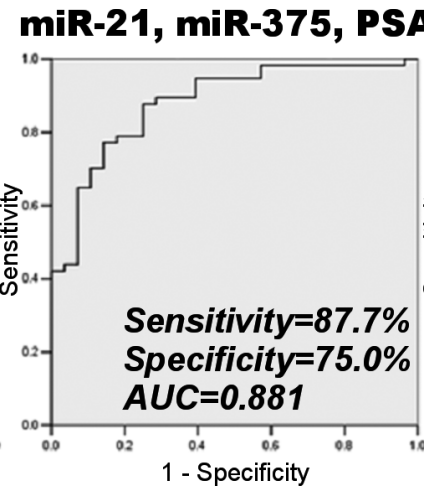

miR-21, PSA

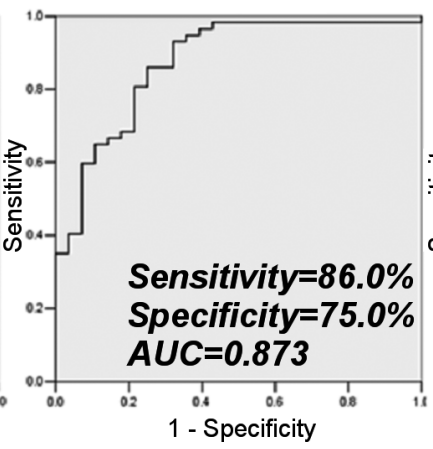

miR-375, PSA

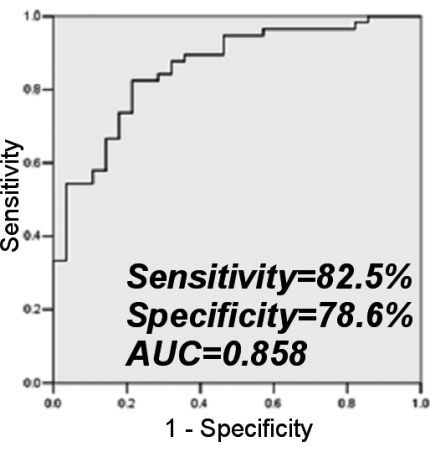

Figure 4. Multimarker ROC curve analysis by combinations of miR-21, miR-375 and prostate-specific antigen (PSA) to differentiate PCa (n=57) from BPH $(n=28)$ controls.

significantly higher than $35 \mathrm{BPH}$ patients (median: 0.360) and 18 healthy controls (median: 0.405) [20]. Haldrup et al. reported that miR-375 level was upregulated by 14.66 -fold in serum samples from $31 \mathrm{PCa}$ patients when compared to 13 BPH control patients $(p=0.009)[21]$. When Kachakova et al. studied the diagnostic potency of let-7c, miR-30c, miR-141, and miR-375, they found that miR-375 showed a stronger diagnostic accuracy of PCa patients compared to $\mathrm{BPH}$ controls than other miRNAs [16]. However, based on their results miR-375 was significantly downregulated in $83.5 \%$ of PCa patients compared to BPH controls. They explained the observed downregulation of miR-375 with the small number of patients with metastatic PCa, and with the possible interference arising from diabetes, allergic diseases and other types of cancers. We intentionally excluded those who had signs of diabetes or inflammation from this study during the enrollment. Hence, our findings suggested that miR-375 was upregulated in $\mathrm{PCa}$ pateints.

From the data of ROC analysis, the AUC of miR-21 and miR-375 were 0.799 and 0.757 respectively. The two studied miRNAs appeared more specific (specificity of $75.0 \%$ for both) than PSA (69.7\%). The sensitivity of miR-21 was $87.7 \%$, close to that of PSA (91.2\%), and higher than miR375 (75.4\%). ROC analysis was used further to evaluate the joint diagnostic value of the two miRNAs and PSA. It was found that PSA in combination with miR-21 and/or miR375 greatly increased the diagnostic specificity. The AUC of the combined miR-21, miR-375 and PSA was the highest (0.881), with sensitivity of $87.7 \%$ and specificity of $75 \%$. The cocktail of miRNA and PSA seemed to have better diagnostic potential than PSA alone. This is consistent with the findings of Kachakova et al., in which the largest AUC was obtained when let-7c, miR-30c, miR-375 and PSA combined together, with sensitivity of $92.7 \%$ and specificity of $66.7 \%$, much more specific than PSA alone (at $4 \mathrm{ng} / \mathrm{ml}$ of PSA, sensitivity was $100 \%$, and the specificity was barely $6.2 \%$ ) [16].

In the present study, the PCa patients can be further grouped into metastatic PCa (N1/M1, 12 patients) and localized PCa (47 patients), based on tumor staging. Using independent-sample Student's t-test, we detected miR-21 expression level was significantly higher in metastatic PCa subgroup than in localized PCa. No statistic difference was observed for miR-375 (data not shown). Spearman analysis also revealed that miR-21 was correlated with Gleason score and tumor staging, while miR-375 not. The relationship of miR-21 and PCa aggressiveness are still controversial. In some studies miR-21 was found to be significantly higher in metastatic PCa group than in localized PCa group [9, 18]. Watahiki Aet al. identified 63 miRNAs upregulated in mCRPC versus localized PCa using Exiqon miRNA qPCR panel, but no miRNA alone differentiated localized PCa and mCRPC [22]. More cohort studies are required for this purpose.

In conclusion, miR-2 1 and miR-375 levels are significantly higher in the plasma of PCa patients than noncancer controls. At the optimal cutoff, miR-21 alone may be a qualified candidate (with sensitivity of $87.7 \%$ and sensitivity of $75.0 \%$ ). The combination of the studied plasma miRNAs and PSA outperforms the miRNAs or PSA testing alone and has the potential to be used as a noninvasive diagnostic cocktail for PC screening.

Acknowledgments: This study was supported by Natural Science Foundation of Liaoning Province of China (No.2013023044) and (2015010289-301), and by International S\&T Cooperation Program of China (2013DFA11150).

\section{References}

[1] SIEGEL R, WARD E, BRAWLEY O, JEMAL A. Cancer statistics, 2011: the impact of eliminating socioeconomic and racial disparities on premature cancer deaths. CA Cancer J Clin 2011; 61: 212-236. http://dx.doi.org/10.3322/caac.20121

[2] MONN MF, TATEM AJ, CHENG L. Prevalence and management of prostate cancer among East Asian men: Current trends and future perspectives. Urol Oncol 2016; 34: 58 e51-59. 
[3] THOMPSON IM, GOODMAN PJ, TANGEN CM, LUCIA MS, MILLER GJ et al. The influence of finasteride on the development of prostate cancer. N Engl J Med 2003; 349: 215-224. http://dx.doi.org/10.1056/NEJMoa030660

[4] ZHANG HL, QIN XJ, CAO DL, ZHU Y, YAO XD et al. An elevated serum miR-141 level in patients with bone-metastatic prostate cancer is correlated with more bone lesions. Asian J Androl 2013; 15: 231-235. http://dx.doi.org/10.1038/ aja.2012.116

[5] KOZOMARA A, GRIFFITHS-JONES S. miRBase: integrating microRNA annotation and deep-sequencing data. Nucleic Acids Res 2011;39: D152-157. http://dx.doi.org/10.1093/nar/ gkq1027

[6] BERTOLI G, CAVA C, CASTIGLIONI I. MicroRNAs: New Biomarkers for Diagnosis, Prognosis, Therapy Prediction and Therapeutic Tools for Breast Cancer. Theranostics 2015; 5: 1122-1143. http://dx.doi.org/10.7150/thno.11543

[7] LU J, GETZ G, MISKA EA, ALVAREZ-SAAVEDRA E, LAMB $J$ et al. MicroRNA expression profiles classify human cancers. Nature 2005; 435: 834-838. http://dx.doi.org/10.1038/ nature 03702

[8] CHEN X, BA Y, MA L, CAI X, YIN Y et al. Characterization of microRNAs in serum: a novel class of biomarkers for diagnosis of cancer and other diseases. Cell Res 2008; 18: 997-1006. http://dx.doi.org/10.1038/cr.2008.282

[9] ZHANG HL, YANG LF, ZHU Y, YAO XD, ZHANG SL et al. Serum miRNA-21: elevated levels in patients with metastatic hormone-refractory prostate cancer and potential predictive factor for the efficacy of docetaxel-based chemotherapy. Prostate 2011; 71: 326-331. http://dx.doi.org/10.1002/ pros.21246

[10] ZHENG Q, PESKOE SB, RIBAS J, RAFIQI F, KUDROLLI T et al. Investigation of miR-21, miR-141, and miR-221 expression levels in prostate adenocarcinoma for associated risk of recurrence after radical prostatectomy. Prostate 2014; 74: 1655-1662. http://dx.doi.org/10.1002/pros.22883

[11] HUANG X, YUAN T, LIANG M, DU M, XIA S et al. Exosomal miR-1290 and miR-375 as prognostic markers in castrationresistant prostate cancer. Eur Urol 2015; 67: 33-41. http:// dx.doi.org/10.1016/j.eururo.2014.07.035

[12] PAPAGIANNAKOPOULOS T, SHAPIRO A, KOSIK KS. MicroRNA-21 targets a network of key tumor-suppressive pathways in glioblastoma cells. Cancer Res 2008; 68: 81648172. http://dx.doi.org/10.1158/0008-5472.CAN-08-1305

[13] RIBAS J, NI X, HAFFNER M, WENTZEL EA, SALMASI AH et al. miR-21: an androgen receptor-regulated microRNA that promotes hormone-dependent and hormone-independent prostate cancer growth. Cancer Res 2009; 69: 7165-7169. http://dx.doi.org/10.1158/0008-5472.CAN-09-1448

[14] YAN JW, LIN JS, HE XX. The emerging role of miR-375 in cancer. Int J Cancer 2014; 135: 1011-1018. http://dx.doi. org/10.1002/ijc.28563

[15] SZCZYRBA J, NOLTE E, WACH S, KREMMER E, STOHR $\mathrm{R}$ et al. Downregulation of Sec23A protein by miRNA-375 in prostate carcinoma. Mol Cancer Res 2011; 9: 791-800. http:// dx.doi.org/10.1158/1541-7786.MCR-10-0573

[16] KACHAKOVA D, MITKOVA A, POPOV E, POPOV I, VLAHOVA A et al. Combinations of serum prostate-specific antigen and plasma expression levels of let-7c, miR-30c, miR141 , and miR-375 as potential better diagnostic biomarkers for prostate cancer. DNA Cell Biol 2015; 34: 189-200. http:// dx.doi.org/10.1089/dna.2014.2663

[17] MITCHELL PS, PARKIN RK, KROH EM, FRITZ BR, WYMAN SK et al. Circulating microRNAs as stable bloodbased markers for cancer detection. Proc Natl Acad Sci U S A 2008; 105: 10513-10518. http://dx.doi.org/10.1073/ pnas.0804549105

[18] YAMAN AGAOGLU F, KOVANCILAR M, DIZDAR Y, DARENDELILER E, HOLDENRIEDER S et al. Investigation of miR-21, miR-141, and miR-221 in blood circulation of patients with prostate cancer. Tumour Biol 2011; 32: 583-588. http://dx.doi.org/10.1007/s13277-011-0154-9

[19] KOTB S, MOSHARAFA A, ESSAWI M, HASSAN H, MESHREF A et al. Circulating miRNAs 21 and 221 as biomarkers for early diagnosis of prostate cancer. Tumour Biol 2014; 35: 12613-12617. http://dx.doi.org/10.1007/s13277-014$\underline{2584-7}$

[20] WACH S, AL-JANABI O, WEIGELT K, FISCHER K, GREITHER T et al. The combined serum levels of miR-375 and urokinase plasminogen activator receptor are suggested as diagnostic and prognostic biomarkers in prostate cancer. Int J Cancer 2015; 137: 1406-1416. http://dx.doi.org/10.1002/ ijc. 29505

[21] HALDRUP C, KOSAKA N, OCHIYA T, BORRE M, HOYER $S$ et al. Profiling of circulating microRNAs for prostate cancer biomarker discovery. Drug Deliv Transl Res 2014; 4: 19-30. http://dx.doi.org/10.1007/s13346-013-0169-4

[22] WATAHIKI A, MACFARLANE RJ, GLEAVE ME, CREA F, WANG Y et al. Plasma miRNAs as biomarkers to identify patients with castration-resistant metastatic prostate cancer. Int J Mol Sci 2013; 14: 7757-7770. http://dx.doi.org/10.3390/ ijms14047757 